\title{
Complement dysregulation is associated with severe COVID-19 illness
}

\section{Jia Yu, Gloria F. Gerber, Hang Chen, Xuan Yuan, Shruti Chaturvedi, Evan M. Braunstein and Robert A. Brodsky}

Division of Hematology, Department of Medicine, Johns Hopkins School of Medicine, Baltimore, MD, USA

\section{ABSTRACT}

S evere acute respiratory syndrome coronavirus-2 (SARS-CoV-2) may manifest as thrombosis, stroke, renal failure, myocardial infarction, and thrombocytopenia, reminiscent of other complement-mediated diseases. Multiple clinical and preclinical studies have implicated complement in the pathogenesis of COVID-19 illness. We previously found that the SARS-CoV-2 spike protein activates the alternative pathway of complement (APC) in vitro through interfering with the function of complement factor $\mathrm{H}$, a key negative regulator of APC. Here, we demonstrated that serum from 58 COVID-19 patients $(32$ patients with minimal oxygen requirement, 7 on high flow oxygen, 17 requiring mechanical ventilation and 2 deaths) can induce complementmediated cell death in a functional assay (the modified Ham test) and increase membrane attack complex (C5b-9) deposition on the cell surface. A positive modified Ham assay ( $>20 \%$ cell-killing) was present in $41.2 \%$ COVID-19 patients requiring intubation ( $n=7 / 17)$ and only $6.3 \%$ in COVID-19 patients requiring minimal oxygen support $(n=2 / 32)$. C5 and factor $\mathrm{D}$ inhibition effectively mitigated the complement amplification induced by COVID-19 patient serum. Increased serum factor Bb level was associated with disease severity in COVID-19 patients, suggesting that APC dysregulation plays an important role. Moreover, SARS-CoV-2 spike proteins directly block complement factor $\mathrm{H}$ from binding to heparin, which may lead to complement dysregulation on the cell surface. Taken together, our data suggest that complement dysregulation contributes to the pathogenesis of COVID-19 and may be a marker of disease severity.

\section{Introduction}

Complement has emerged as a potential driver of the pathogenesis of severe coronavirus disease 2019 (COVID-19). ${ }^{1-3}$ Clinically, endothelial damage, systemic microvascular thrombosis and recalcitrant hypercoagulability observed in COVID19 mirror other disorders of complement regulation, such as atypical hemolytic uremic syndrome (aHUS) and catastrophic antiphospholipid antibody syndrome (CAPS). ${ }^{1-6}$ Autopsy studies showed microvascular thrombi and complement deposition in the lungs and kidneys of patients with severe COVID-19.,8 Several studies have demonstrated elevated levels of soluble C5a and C5b-9 in COVID-19 patients. ${ }^{9,10}$ Further, plasma C3a levels are higher in COVID-19 patients admitted to the intensive care unit (ICU) compared to non-ICU patients. ${ }^{11}$ A small number of patients treated with eculizumab, a monoclonal anti-C5 antibody, experienced rapid improvement in hypoxia and inflammatory markers. ${ }^{12,13}$ Three patients treated with an upstream complement C3 inhibitor demonstrated reduction in inflammatory markers and improved disease severity within 48 hours of treatment. ${ }^{14} \mathrm{Clinical}$ trials of complement inhibitors in COVID-19 are ongoing (clinicaltrials gov. Identifier: NCT04355494, NCT04390464).

The complement system, a key component of the human innate immune response, is a collection of proteases, receptors and inhibitors that functions to eliminate cellular debris, promote inflammation and defend against pathogens. ${ }^{15}$ The
Haematologica 2022

Volume 107(5):1095-1105

\section{Correspondence:}

ROBERT A. BRODSKY

brodsro@jhmi.edu

Received: May 17, 2021.

Accepted: July 14, 2021.

Pre-published: July 22, 2021.

https://doi.org/10.3324/haematol.2021.279155

(C)2022 Ferrata Storti Foundation

Material published in Haematologica is covered by copyright. All rights are reserved to the Ferrata Storti Foundation. Use of published material is allowed under the following terms and conditions:

https://creativecommons.org/licenses/by-nc/4.0/legalcode. Copies of published material are allowed for personal or internal use. Sharing published material for non-commercial purposes is subject to the following conditions:

https://creativecommons.org/licenses/by-nc/4.0/legalcode, sect. 3. Reproducing and sharing published material for commercial purposes is not allowed without permission in writing from the publisher. 
system consists of three major pathways - the classical, lectin and alternative pathways (APC). The three pathways converge on the cleavage of $\mathrm{C} 3$ and subsequently $\mathrm{C} 5$, which produces anaphylatoxins (C3a and C5a, respectively) that are associated with the acute inflammatory response and thrombosis. ${ }^{16}$ The membrane attack complex (C5b-9) is the terminal product of complement activation. ${ }^{15}$

Evidence for the role of complement in COVID-19 continues to emerge, but understanding of the underlying mechanisms remains incomplete. Recently, we demonstrated that the SARS-CoV-2 spike protein (subunits 1 and 2) converts inactivator surfaces into activator surfaces through interference with the function of complement factor $\mathrm{H}(\mathrm{CFH})$, a critical negative regulator of APC on host cells. ${ }^{17}$ These studies were performed by adding recombinant spike protein to normal human serum (NHS) and measuring complement-mediated killing on the surface of nucleated cells. Complement inhibition with C5 and factor $\mathrm{D}$ inhibitors effectively prevented C $5 b-9$ accumulation induced by the SARS-CoV-2 spike proteins. ${ }^{17}$ In order to confirm that complement is a rational target for treating COVID-19, it is important to show that serum from patients infected with SARS-CoV-2 also displays complement dysregulation. In this study, we evaluated cell surface C5b-9 deposition and complement-mediated cell killing induced by COVID-19 patient sera in the modified Ham (mHam) test ${ }^{5,18,19}$ and showed that impaired complement regulation correlates with COVID-19 disease severity and can be mitigated with complement inhibitors.

\section{Methods}

\section{Patients and samples collection}

Serum samples and a limited clinical data set were obtained for 58 COVID-19 patients from the Clinical Characterization Protocol for Severe Infectious Diseases repository (clinicaltrials gov. Identifier: NCT04496466), approved by the Johns Hopkins Institutional Review Board. Samples were collected between April and May 2020. One sample collected outside of the patient's hospitalization for COVID-19 was excluded. We also recruited one COVID-19 patient requiring mechanical ventilation and five healthy individuals who received the Pfizer-BioNTech (BTN162b2) COVID-19 vaccine between November and December 2020. Informed written consent for sample use from the Complement Associated Disorders Registry study was obtained. Blood was collected by venipuncture in serum separator tubes.

Date of COVID-19 diagnosis was based on the earliest known positive SARS-CoV-2 nucleic acid amplification test from a nasopharyngeal swab or time an infection flag was placed on the patient's chart. Severity of COVID-19 was graded based on World Health Organization (WHO) eight-point ordinal outcome scale. ${ }^{20}$

\section{The modified Ham test}

The mHam test was used to assess complement-mediated cell killing of TF1PIGAnull cells induced by patient serum as previously described..$^{5,17-19,21}$ The assay is detailed in the Online Supplementary Appendix.

\section{Detection of complement activity by flow cytometry}

C5b-9 and C3c deposition after incubation of TF1PIGAnull cells with COVID-19 patient serum was measured by flow cytometry as previously described..$^{5,17,21}$ The assay is detailed in Online Supplementary Appendix.

\section{Complement inhibition in patient serum}

Patient serum was incubated with $1 \mu \mathrm{M}$ factor D inhibitor (ACH145951, Achillion Pharmaceuticals) or $50 \mu \mathrm{g}$ anti-C5 monoclonal antibody (anti-C5Ab, Alexion pharmaceuticals) and then added to cells. C5b-9 and C3c deposition was measured by flow cytometry.

\section{Quantification of serum factor Bb by enzyme-linked immunosorbant assay}

Factor $\mathrm{Bb}$ level in patient serum was measured using MicroVue Bb Plus EIA kit (Quidel). NHS preincubated with $20 \mu \mathrm{g} / \mathrm{mL}$ cobra venom factor (Complement Technology) served as a positive control.

\section{Competitive heparin binding assay}

Immunoprecipitation of His-tagged SARS-CoV-2 spike protein subunit 1 (S1, RayBiotech) and subunit 2 (S2, RayBiotech) was performed using Heparin-Sepharose beads (BioVision). We incubated $0.2 \mathrm{M} \mathrm{S} 1$ or S2 overnight at $4^{\circ} \mathrm{C}$ with $80 \mu \mathrm{L}$ beads in the presence and absence of $0.2 \mathrm{M} \mathrm{CFH}$ protein (Complement Technology) in phosphate buffered saline. After washing, the beads were denatured with LDS buffer (Invitrogen) and reducing agent (Invitrogen) at $70^{\circ} \mathrm{C}$ for 15 minutes and then centrifuged. The supernatants were collected for western blotting. We used 10 $\mu \mathrm{L}$ of input protein solution for western blotting.

We loaded $10 \mu \mathrm{L}$ reduced proteins to mini-PROTEIN TGX Gels (Bio-Rad Laboratories) and then transferred to PVDF membranes. The membrane was probed with anti-CFH antibody $(1: 1,500$, Cat. Sc-53073, Santa Cruz Biotechnology) or anti-His antibody (1:2,000, Cat. Sc-53073, Santa Cruz Biotechnology). The blot was incubated with HRP-linked anti-mouse immunoglobulin (Ig) G (1:10,000, Cat. 7076S, Cell Signaling Technology) and imaged with SuperSignal West Femto Maximum Sensitivity Substrate (Thermo Fisher).

\section{Statistical analysis}

Data was summarized as mean \pm standard error (SE). $\chi^{2}$ test was used to compare the rates of mHam positivity across WHO groups. Student's $t$-test was used to evaluate differences between unpaired groups. $P<0.05$ was considered statistically significant.

\section{Results}

\section{Patient characteristics}

All 58 patients studied required hospitalization for their COVID-19 illness (WHO score 4-8). Thirty-two patients required minimal oxygen support (WHO score 4), seven required high flow nasal cannula oxygen therapy (WHO score 5), 17 received mechanical ventilation and additional organ support (WHO score 7), and two died (WHO score 8) (Table 1). Five healthy individuals were recruited at the same hospital system and their serum samples post COVID-19 vaccination were tested for complement activation.

\section{COVID-19 patient serum induced complement-mediated cell killing}

The mHam measures the ability of a nucleated cell to protect itself from complement-mediated cell killing in vitro in the absence of downstream cell-surface complement regulators, CD55 and CD59. ${ }^{18,21}$ Thus, mHam exposes defects in complement regulation in patient serum. This assay has been validated in complement-mediated disorders such as aHUS, HELLP and CAPS. ${ }^{5,18,21,22}$ The mHam 
Table 1. COVID-19 patient characteristics.

\begin{tabular}{|c|c|c|c|c|}
\hline & $\begin{array}{l}\text { Oxygen } \\
(\mathrm{N}=32)\end{array}$ & $\begin{array}{l}\text { Hi-Flow Oxygen } \\
\qquad(\mathrm{N}=7)\end{array}$ & $\begin{array}{l}\text { Ventilation } \\
\qquad(\mathrm{N}=17)\end{array}$ & $\begin{array}{l}\text { Death } \\
(\mathrm{N}=2)\end{array}$ \\
\hline Age (years), median & $55(26-78)$ & $58(49-69)$ & $59(27-80)$ & $57(56-57)$ \\
\hline \multicolumn{5}{|l|}{ Sex } \\
\hline Female & 18 & 3 & 7 & 1 \\
\hline Male & 14 & 4 & 10 & 1 \\
\hline \multicolumn{5}{|l|}{ Race } \\
\hline Black & 11 & 4 & 9 & 1 \\
\hline White & 11 & 2 & 4 & 0 \\
\hline Other & 10 & 1 & 4 & 1 \\
\hline \multicolumn{5}{|l|}{ Ethnicity } \\
\hline Hispanic & 23 & 6 & 14 & 1 \\
\hline Non-hispanic & 9 & 1 & 3 & 1 \\
\hline BMI, median & $33.6(20.5-58.2)$ & $34.2(27.1-51.9)$ & $30.4(16.2-46.3)$ & 65.3 \\
\hline $\begin{array}{l}\text { Days from COVID-19 diagnosis } \\
\text { to serum sample collection, median }\end{array}$ & $4(1-13)$ & $8(2-16)$ & $13(3-34)$ & 18 \\
\hline $\begin{array}{l}\text { Days from hospital admission } \\
\text { to serum sample collection, median }\end{array}$ & $2(1-13)$ & $6(1-10)$ & $13(2-32)$ & 13 \\
\hline Length of hospitalization (days), median & $9(2-45)$ & $11(7-15)$ & $28(3-70)$ & 17 \\
\hline
\end{tabular}

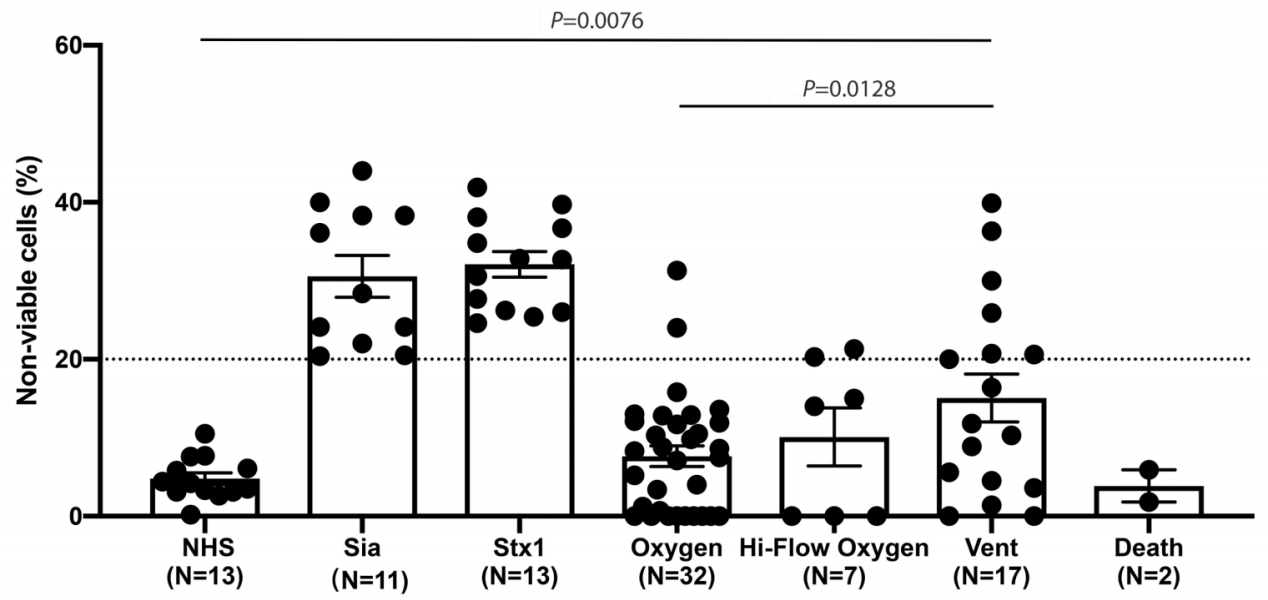

Figure 1. Complement-mediated cell killing induced by COVID-19 patient serum is associated with disease severity. TF1PIGAnull cells were treated with 20\% COVID19 patient serum, and cell killing was measured using the modified Ham (mHam) test. Complement-mediated cell killing (\%) was significantly elevated in COVID-19 patients requiring mechanical ventilation (vent), as compared to pooled normal human serum (NHS) and COVID-19 patients who needed minimal oxygen support $(P<0.01)$. The dotted line at $20 \%$ non-viable cells represented the threshold for a positive mHam based on a previously established receiver operator curve. All experiments were run in triplicate. Sia: sialidase (used as a positive control); stx1: Shiga toxin subunit 1 (used as a positive control). Hi-Flow: High-Flow Oxygen.

test was positive (> 20\% cell killing) in $41.2 \%$ ( 7 of 17 ) of patients who required intubation (WHO score 7 ), compared to $6.3 \%$ (2 of 32 ) of those who only needed minimal oxygen support (WHO score 4$)(P=0.002)$ (Figure 1). Serum from COVID-19 patients who required mechanical ventilation induced significantly higher cell killing compared to those on minimal oxygen support (mean $17.3 \%$ vs. $7.7 \%$, $P<0.01$ ), suggesting that SARS-CoV-2 infection impairs the ability to regulate complement on cells.

\section{COVID-19 patient serum increased C5b-9 and C3c deposition on cell surface}

C3c and C5b-9 deposition, biomarkers for complement activation, on the surface of TF1PIGAnull cells after exposure to COVID-19 patient serum was evaluated. All com- plement pathway buffer $\left(\mathrm{GVB}^{++}\right)$and alternative pathway specific buffer (GVB ${ }^{0} \mathrm{MgEGTA}$ ) were used. In all pathway buffer, C5b-9 deposition was increased compared to control normal human serum in virtually all COVID-19 patients regardless of WHO score (Figure 2A). In APC specific buffer, the deposition of C5b-9 was significantly elevated in patients requiring minimal oxygen support, intubation and those who died (Figure 2B) compared to control serum. C3c deposition was significantly increased in all patient groups in APC specific buffer (Figure 2D) but not in all complement pathway buffer (Figure 2C).

We selected patient samples with positive mHam and increased C5b-9 deposition to perform blocking experiments. Representative examples of blocking with the complement inhibitors in two patient sera are shown in 
Figure 3. C5b-9 deposition was completely inhibited by blocking the terminal complement pathway with an antiC5 antibody. The factor D inhibitor (ACH145951), an APC-specific inhibitor, partially reduced C5b-9 deposition induced by serum from patient 2 and achieved complete inhibition in patient 1 (Figure 3A). In addition, the factor $\mathrm{D}$ inhibitor was more effective than anti-C5 antibody in inhibiting C3c deposition triggered by COVID-19 patient sera (Figure $3 \mathrm{~B}$ ), since it targets upstream of the anti-C5 monoclonal antibody.
A

All complement pathway buffer

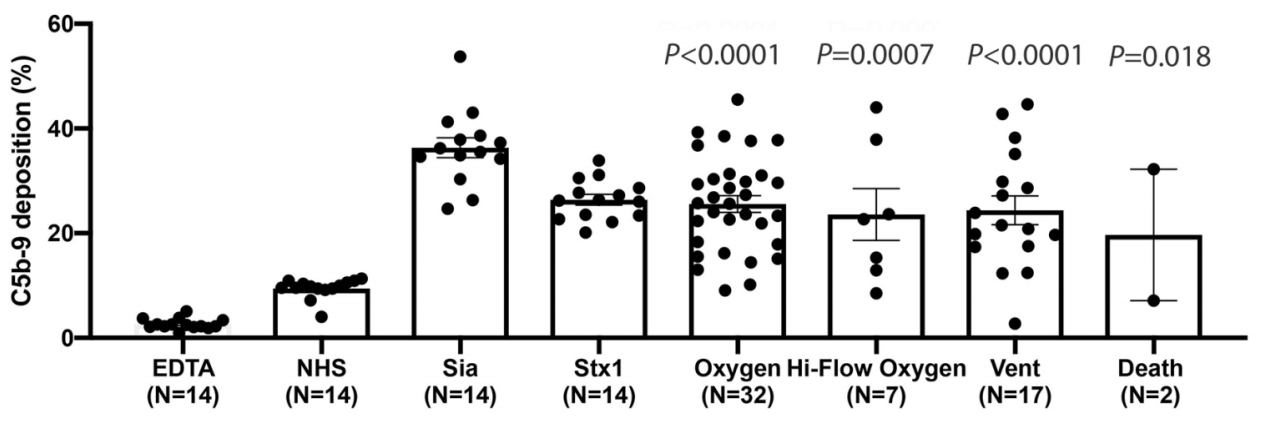

B

APC specific buffer

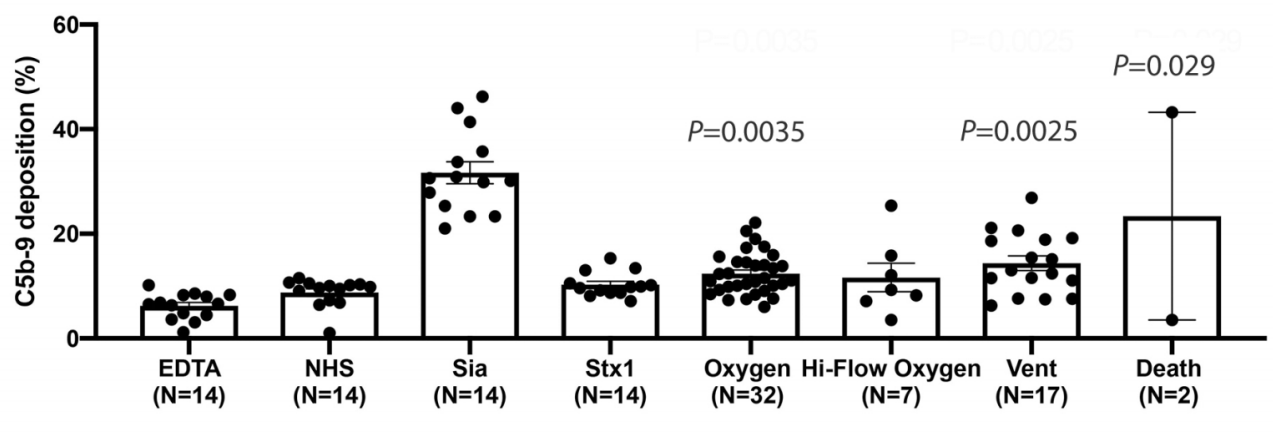

C

All complement pathway buffer
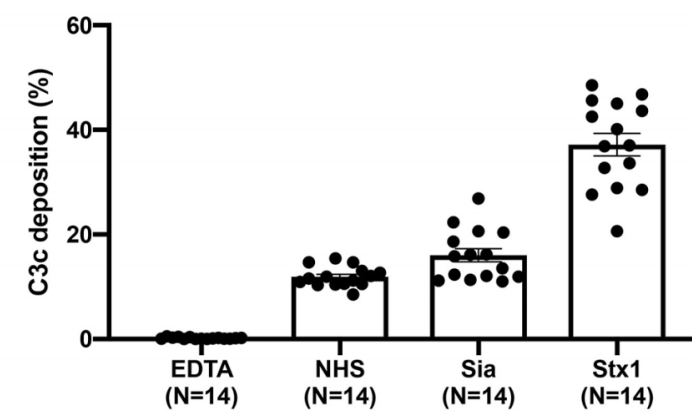

$P=0.023$

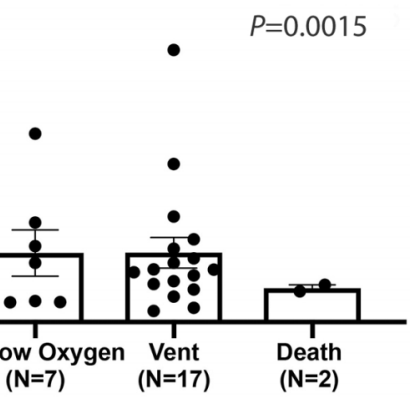

D

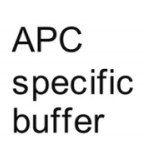

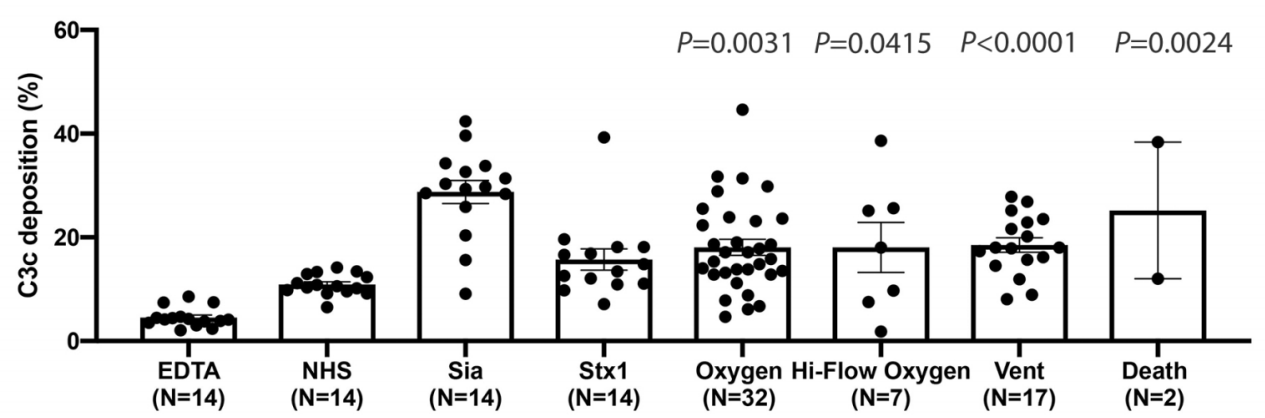

Figure 2. COVID-19 patient serum induces C5b-9 and C3c deposition on the cell surface. COVID-19 patient serum led to increased cell surface C5b-9 deposition in all complement pathway buffer (A) and alternative pathway specific buffer (B). C3c deposition was increased compared to control normal human serum (NHS) in all complement pathway buffer (C) and alternative pathway specific buffer (D). Statistical significance was calculated between each disease group and the NHS control group. EDTA: ethylenediaminetetraacetic (used as a negative control); Sia: sialidase (used as a positive control for alternative pathway of complement [APC] activation); stx1: Shiga toxin subunit 1 (used as a positive control for all complement pathways activation). Vent: ventilation. 
A

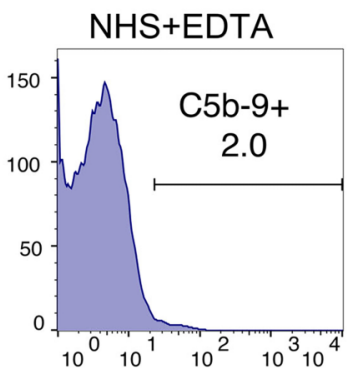

Patient 1

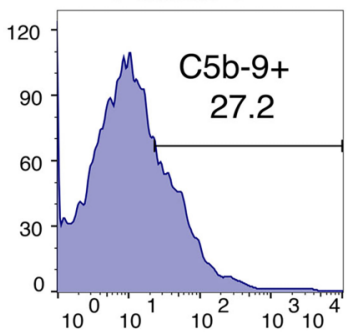

Patient 2

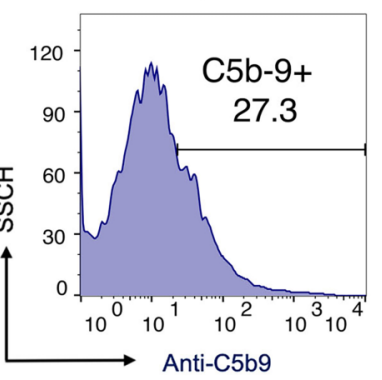

NHS+EDTA

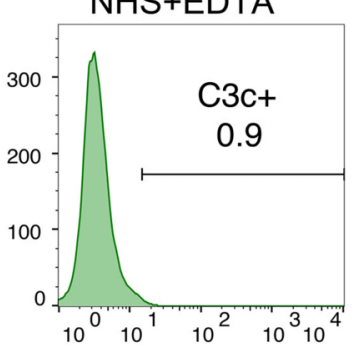

Patient 1

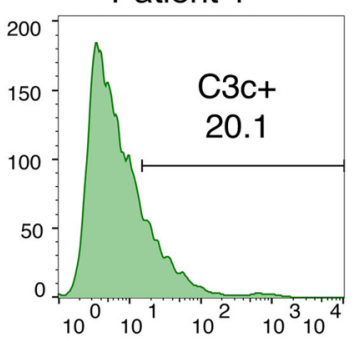

Patient 2

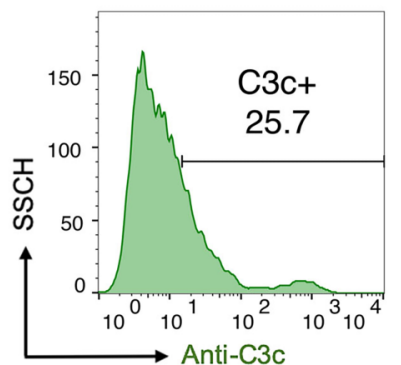

NHS

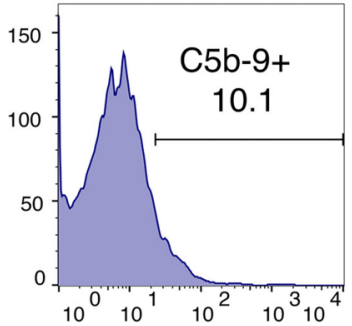

Pt 1+ACH145951

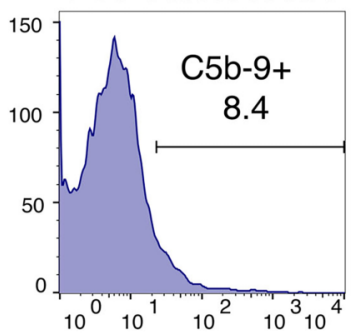

Pt 2+ACH145951

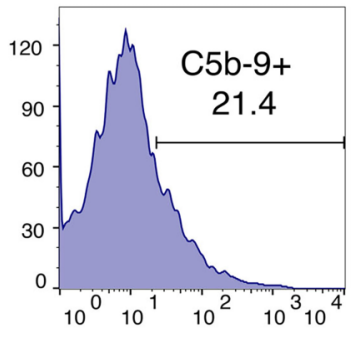

NHS

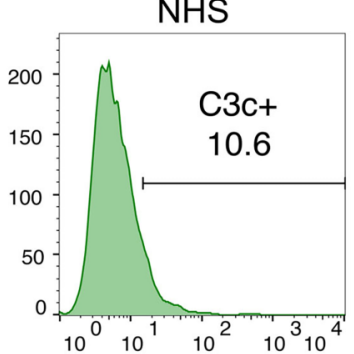

Pt 1+ACH145951

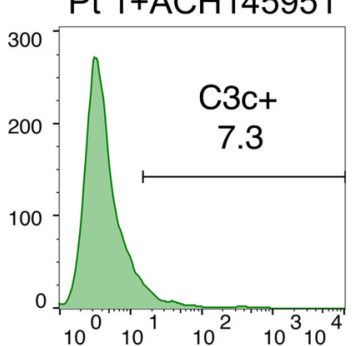

Pt 2+ACH145951

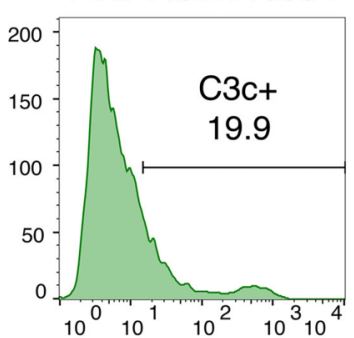

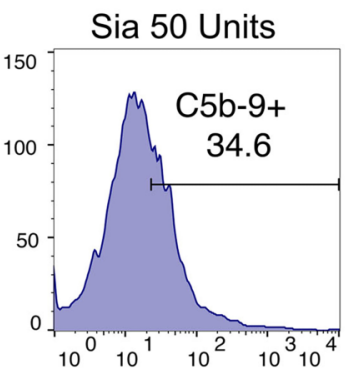

Pt 1+Anti-C5 Ab

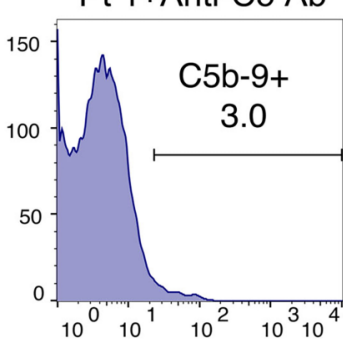

Pt 2+Anti-C5 Ab
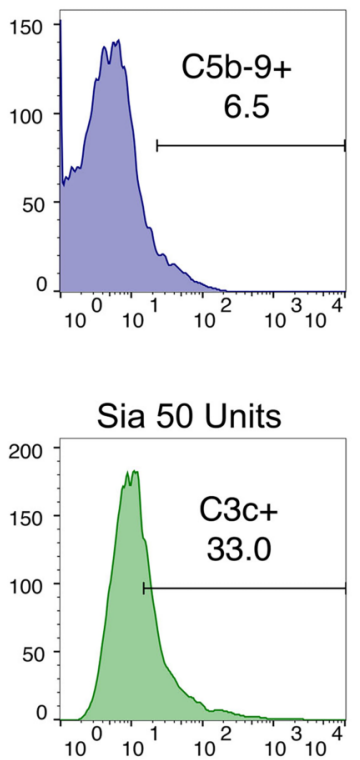

Pt 1+Anti-C5 Ab

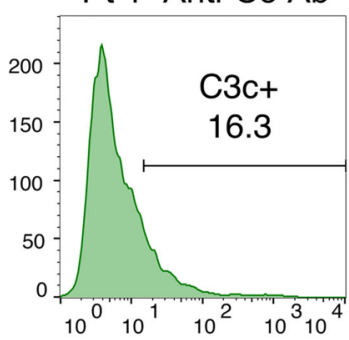

Pt 2+Anti-C5 Ab

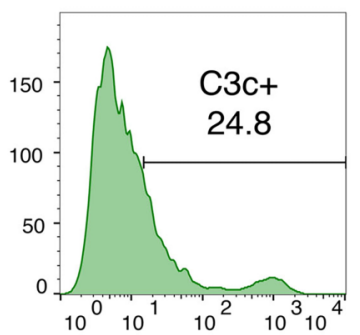

Figure 3. C5 and factor D inhibition block complement activation induced by COVID-19 patien serum. Flow cytometry demonstrated increased C5b-9 (A) and C3c (B) deposition in two representative COVID-19 patients. C5b-9 deposition was completely blocked in the presence of $50 \mu \mathrm{g}$ anti-C5 antibody. $1 \mathrm{uM}$ factor D inhibitor (ACH145951) partially reduced the C5b-9 deposition in patient 2 and achieved complete inhibition in patient 1 (A). Factor D inhibitor also effectively decreased C3c accumulation induced by both patients sera, whereas anti-C5 antibody did not appreciably prevent C3c deposition (B). SSCH: side scatter ACH145951: factor D inhibitor; AntiC5 Ab: anti-C5 monoclonal antibody. NHS: normal human serum; EDTA: ethylenediaminetetraacetic 


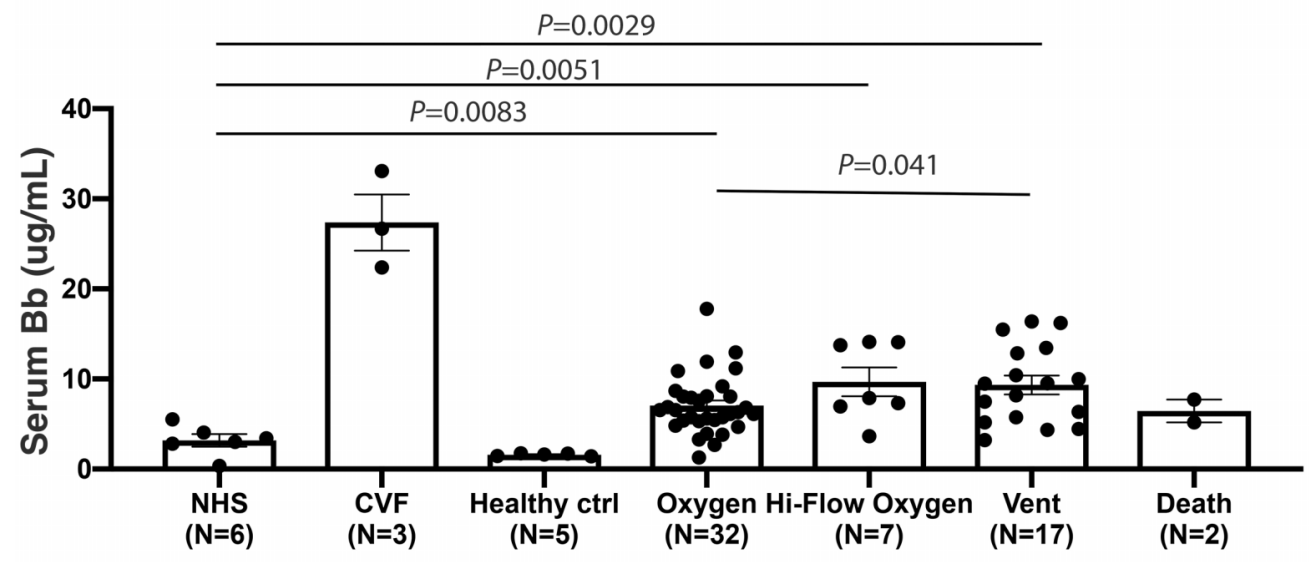

Figure 4. The Bb level is increased in COVID-19 patient serum and associated with disease severity. Serum Bb level was significantly elevated in COVID-19 patients who required minimal oxygen support, high flow nasal cannula oxygen therapy (Hi-Flow oxygen) and mechanical ventilation (Vent) as compared to pooled normal human serum (NHS) and healthy controls (healthy $\mathrm{ctrl}$ ). Patients requiring mechanical ventilation also had significantly higher serum Bb level than patients with minimal oxygen support $(P<0.05)$, suggesting greater alternative pathway of complement dysregulation. All experiments were run in duplicate. CVF: cobra venom factor (used as a positive control).

Increased alternative pathway of complement activation is associated with COVID-19 disease severity

We utilized standard enzyme-linked immunosorbant assay (ELISA) to measure the factor Bb level in COVID-19 patient serum. Factor $\mathrm{Bb}$, which results from cleavage of factor B by factor D, is a biomarker of APC activation. Regardless of disease severity, the serum level of $\mathrm{Bb}$ was significantly higher in COVID-19 patients compared to the healthy controls. We also found that COVID-19 patients requiring intubation (WHO score 7) had significantly higher $\mathrm{Bb}$ levels than those requiring minimal oxygen support (WHO score 4) (Figure 4). These observations suggested that increased APC activation is associated with disease severity in COVID-19.

\section{SARS-CoV-2 spike proteins compete with complement factor $\mathrm{H}$ for cell surface heparan sulfate binding}

We previously demonstrated that SARS-CoV-2 spike proteins (both subunit 1 and 2) bind heparan sulfate on the cell surface. ${ }^{17}$ Heparan sulfate also serves as a necessary cofactor for binding of SARS-CoV-2 spike proteins to the angiotensin receptor 2 (ACE2) ${ }^{23} \mathrm{CFH}$, a negative regulator of APC, also utilizes glycosaminoglycans, such as heparan sulfate, and $\alpha 2,3 \mathrm{~N}$-linked sialic acid residues for binding to nucleated cells; thus, we hypothesized that SARS-CoV-2 spike protein competes with CFH for binding to heparan sulfate and its tissue specific, more highly-sulfated variant, heparin. In order to evaluate whether SARS-CoV-2 spike proteins block CFH from binding to heparan sulfate, we compared the heparin-binding activity of CFH in the presence and absence of the SARS-CoV-2 spike proteins using heparin-linked beads. CFH alone bound to the heparinbeads with high affinity (Figure $5 \mathrm{~A}$, lane 1 ). In the presence of SARS-CoV-2 S1 and S2, binding of CFH to the heparinbeads was markedly reduced (Figure 5A, lanes 2 and 3 ).

We also compared the heparin-binding ability of SARSCoV-2 spike proteins in the presence and absence of CFH. $\mathrm{S} 1$ alone showed strong binding to heparin-beads and retained high heparin-binding activity in the presence of CFH (Figure 5B). S2 bound to heparin-beads with similarly high efficiency under both conditions (Figure 5C). These results indicated the SARS-CoV-2 spike proteins have high- er binding affinity for heparin than $\mathrm{CFH}$ and interfere with the binding of CFH to heparin.

\section{SARS-CoV-2 mRNA vaccine does not markedly increase complement activity in healthy individuals}

The mRNA COVID-19 vaccines employ the SARS-CoV2 spike protein as an immunogenic target. Given that SARS-CoV-2 spike proteins activate complement in vitro, concern arises whether the COVID-19 vaccine could also trigger transient complement dysregulation in vivo through generation of the spike protein.

In order to test this, we obtained serum samples from five healthy individuals who received both doses of the Pfizer-BioNTech (BTN162b2) COVID-19 vaccine at three time points: before the vaccine (baseline), 24 to 48 hours after receiving the first vaccine dose, and 24 to 48 hours after the second dose. We measured the serum level of factor $\mathrm{Bb}$ in these individuals pre- and post-COVID-19 vaccination. Two of five individuals showed significantly higher serum $\mathrm{Bb}$ levels from their baseline after receiving the COVID-19 vaccine (Figure 6). Notably, these two individuals experienced side effects post vaccination including fever, headache and fatigue. We next performed functional assays to assess for cell surface complement amplification post-vaccination. On average, serum collected after the first vaccine dose did not lead to increased C5b-9 deposition on the surface of TF1PIGAnull cells compared to the individual's serum pre-vaccination. The second dose of the vaccine led to an $11 \%$ increase in the C5b-9 deposition. All individuals had negative mHam results at baseline, which remained negative after receiving the COVID-19 vaccine (data not shown). These results from the functional assays demonstrate that the Pfizer-BioNTech SARS-CoV-2 mRNA vaccine does not sufficiently alter complement regulation in healthy individuals.

\section{Discussion}

Previously, we showed that the SARS-CoV-2 spike protein dysregulates the alternative complement pathway in vitro by interfering with CFH function on the cell. ${ }^{17}$ Here, 
we extend these findings and demonstrate that the SARSCoV-2 spike protein directly competes with CFH for binding to heparan sulfate. In addition, we demonstrate, for the first time, that COVID-19 patient serum induces complement dysregulation on the cell surface using a functional assay (the mHam). Moreover, a positive mHam is highly associated with disease severity in COVID-19 patients, and C5b-9 cell deposition induced by patient sera is blocked by factor D and C5 inhibitors.

Our finding that complement dysregulation is inherent to the pathogenesis of COVID-19 is in agreement with existing preclinical and clinical data. Autopsy studies revealed depositions of complement proteins in lung and other tissues co-localize with the SARS-CoV-2 spike proteins. ${ }^{8,24}$ Markers of complement activation in sera from COVID-19 hospitalized patients are associated with respiratory failure. Specifically, markers of classical/lectin (C4d), alternative (C3bBbP) and common pathway (C3bc, sC5b-9) amplification were increased in COVID-19 patient sera throughout hospitalization, indicative of sustained activation of all complement pathways. ${ }^{9-11,25}$ Sinkovits et al. ${ }^{26}$ showed that complement overactivation and consumption is predictive of in-hospital mortality in SARS-CoV-2 infection. Specifically, these authors reported consumption of C3 in the serum of patients with severe COVID-19 disease. This is consistent with our finding of increased C3c deposition on TF1PIGAnull cells. In transcriptome analysis, expression of multiple complement genes such as C2, C3, CFB and CFH were upregulated in COVID-19 patients. ${ }^{27,28}$ Gavriilaki et al..$^{29}$ analyzed genetic

A

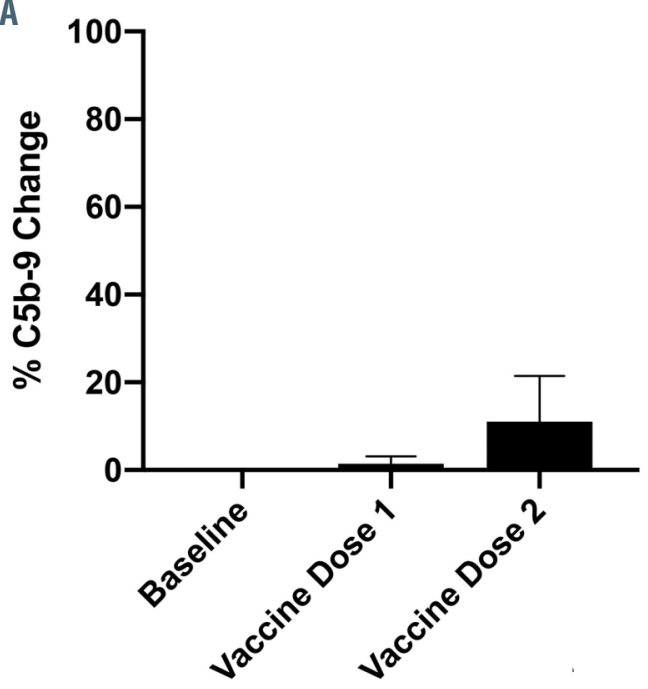

A

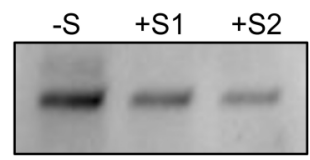

Input -

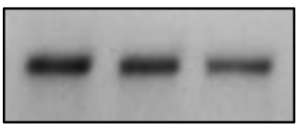

B

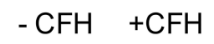

S1 -

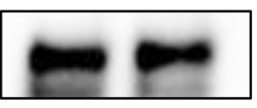

Input -

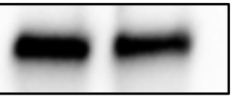

C

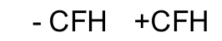

S2 -

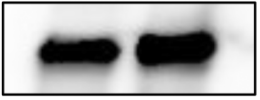

Input -

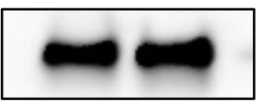

Figure 5 (right). SARS-CoV-2 spike proteins compete with complement factor $\mathrm{H}$ for the same binding sites on heparin. (A) The binding of complement factor $\mathrm{H}$ (CFH) to heparin-linked beads was markedly reduced in the presence of SARSCoV-2 spike protein subunit 1 (S1) and subunit 2 (S2). The western blot was performed using anti-CFH antibody. (B) S1 and S2 retained similarly high binding affinity to heparin-linked beads in the presence and absence of CFH. The western blot was performed using anti-His antibody. The input is the protein solution that is used to incubate with the beads. S: SARS-CoV-2 spike protein; S1: SARSCoV-2 spike protein subunit 1; S2: SARS-CoV-2 spike protein subunit 2.

B

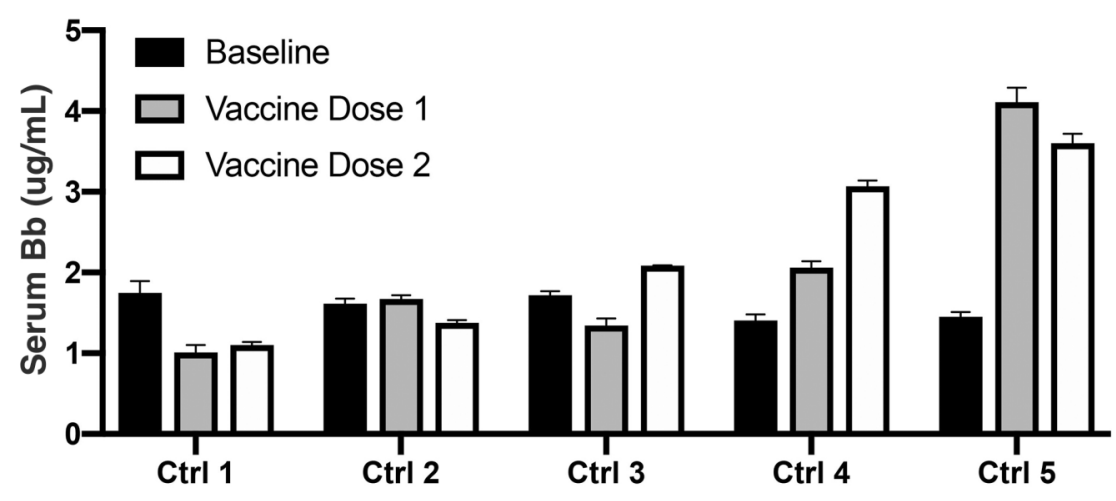

Figure 6. SARS-CoV-2 mRNA vaccine minimally induces cell surface $\mathrm{C5b}-9$ deposition but increases serum Bb level in healthy individuals. Sera from five healthy individuals were collected before they received the COVID-19 mRNA vaccine, $24-48$ hours after their first vaccine dose and $24-48$ hours after their second dose. (A) On average, the first vaccine dose did not lead to elevation in C5b-9 deposition from the individual's baseline, whereas the second dose increased cell surface C5b-9 deposition by $11 \%$. (B) Two of five healthy individuals (Ctrl) had markedly increased serum $\mathrm{Bb}$ level post vaccination as compared to baseline, which correlated with their vaccine side effects including headache, fatigue and fever. 
and clinical data from 97 patients hospitalized with COVID-19 and found an increase in rare variants associated with thrombotic microangiopathies (several involving the alternative pathway of complement) in patients with severe COVID-19 disease.

Complement amplification in hospitalized patients with COVID-19 is multifactorial. Our previous data adding recombinant spike protein to normal human serum showed that complement-mediated cell killing was almost entirely through the alternative complement pathway. ${ }^{17}$ Here, using serum from patients with COVID-19, we find additional contributions from the non-APC pathways. This may be because serum samples in this study were obtained a median of 7 days after testing positive for
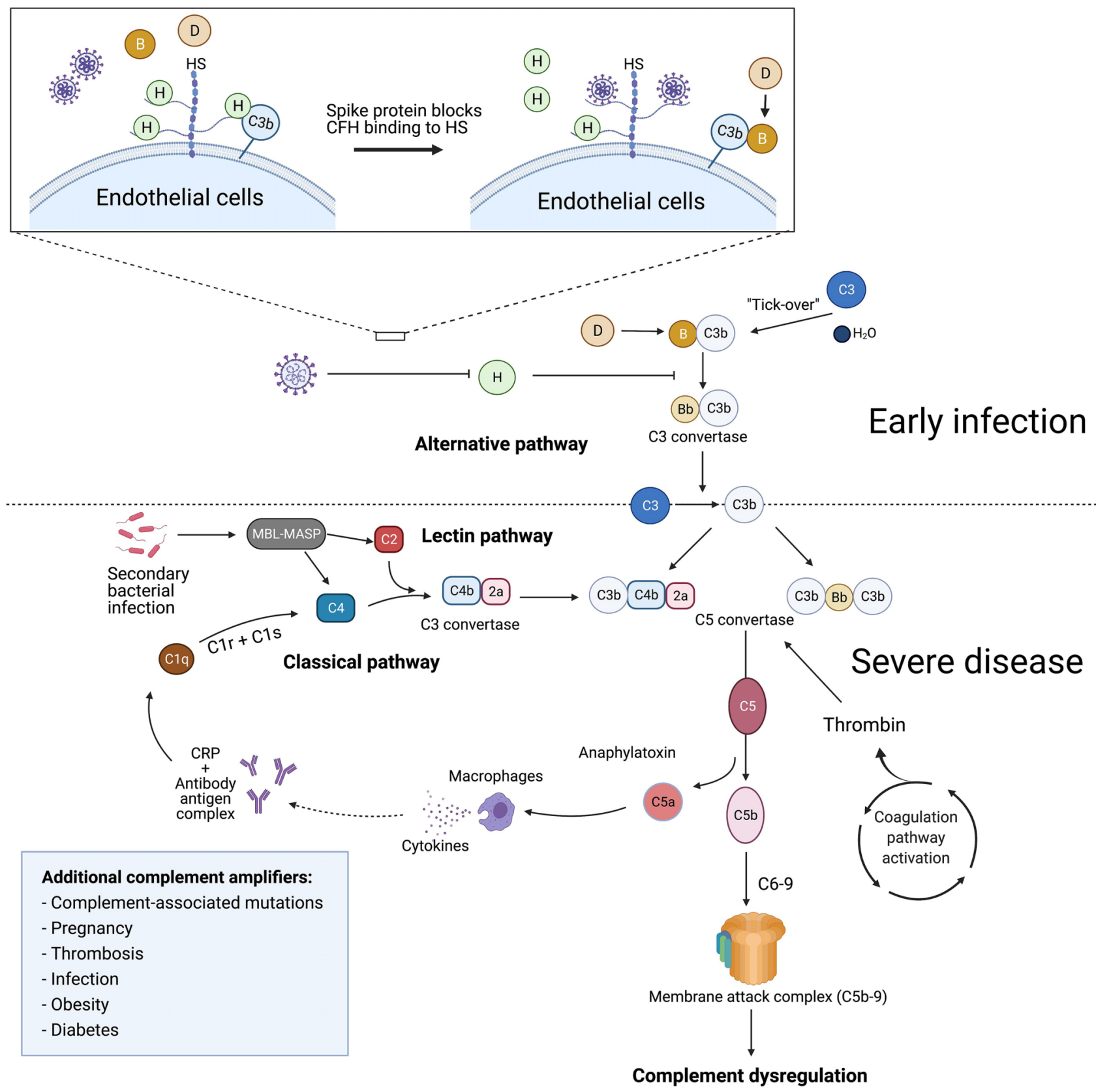

Figure 7. Proposed model for complement dysregulation in SARS-CoV-2 infection. Early in infection, the SARS-CoV-2 spike protein binds heparan sulfate on the endothelial cell surface and interferes with the inhibitory function of complement factor $\mathrm{H}(\mathrm{CFH})$, leading to alternative pathway of complement dysregulation. Suppression of CFH binding results in increased cleavage of factor B by factor $\mathrm{D}$ and generation of $\mathrm{Bb}$. Factor $\mathrm{Bb}$ binds to $\mathrm{C} 3 \mathrm{~b}$ to form the alternative pathway $\mathrm{C} 3$ convertase ( $\mathrm{C} 3 \mathrm{bBb})$, leading to the cleavage of $\mathrm{C} 3$ and generation of the $\mathrm{C} 5$ convertase (C4b2a3b or $\mathrm{C} 3 \mathrm{bBb} 3 \mathrm{~b})$. The $\mathrm{C} 5$ convertase cleaves $\mathrm{C} 5$ to generate $\mathrm{C} 5 \mathrm{a}$ and C5b, which complexes with C6-9 to form the membrane attack complex (C5b-9). C3a and C5a are anaphylatoxins that recruit inflammatory cells and upregulate the expression of acute phase proteins, such as C-reactive protein. Complement amplification from the classical and lectin pathways follows subsequent tissue damage. secondary infections and thromboses. Formation of antibody-antigen complexes can activate the classical complement pathway by binding to the $\mathrm{C} 1$ complex and cleaving $\mathrm{C} 4$ and $\mathrm{C} 2$ to form the classical $\mathrm{C} 3$ convertase (C4b2a). In the lectin pathway, MBL-MASP binds to carbohydrates on the surface of microbes and mediates the cleavage of $\mathrm{C} 2$ and $\mathrm{C} 4$, to generate the $\mathrm{C} 3$ convertase (C4b2a). Preconditions that enhance inflammation (e.g., obesity, diabetes, and vascular disease) or contribute to complement activation (e.g., third-trimester pregnancy) or dysregulation (age-related macular degeneration and other germline complement mutations) may contribute to a severe phenotype through upregulation of these pathways. HS: heparan sulfate; MBL-MASP: mannose-binding protein-mannose-binding lectin serine protease; CRP: C-reactive protein. 
COVID-19, at a time when the SARS-CoV-2 viral load is decreasing, ${ }^{3,31}$ as opposed to measuring cell surface C5b-9 deposition within minutes after adding spike protein to normal human serum. We also demonstrated that mHam positivity (measuring the activation of all complement pathways) from SARS-CoV-2 infected patients is associated with the need for mechanical ventilation; however, we did not find as strong a correlation between cell surface C5b-9 and the mHam as we did after supplementing normal human serum with the spike protein.

Virtually all patients had elevated levels of $\mathrm{Bb}$ in their serum, even two of the healthy subjects after COVID-19 mRNA vaccination, suggesting that APC activation is an early event in the pathogenesis of SARS-CoV-2 infection. This is in agreement with the prominent role for APC found in proteomics studies, which showed increased complement factor B (CFB) levels in serum from severe COVID-19 patients. ${ }^{32}$ CFB deposition was also observed in the lung tissue of COVID-19 patients, and a CFB inhibitor blocked the C3a generated by infection of respiratory epithelial cells with SARS-CoV-2. ${ }^{33,34}$ Pekayvaz et al. ${ }^{35}$ further showed upregulation of complement factor $\mathrm{D}$ (CFD), produced mainly in adipocytes, in monocytes of severe COVID-19 patients. Ma et al. ${ }^{36}$ demonstrated that enhanced activation of the APC is associated with markers of endothelial injury and hypercoagulability in severe COVID-19 patients as compared to other non-COVID-19 patients admitted to the intensive care unit with acute respiratory failure.

C5b-9 deposition induced by COVID-19 patient serum is blocked by both a terminal complement inhibitor (antiC5 antibody) and an alternative pathway specific inhibitor (factor D inhibitor, ACH145951). The factor D inhibitor was more effective in blocking C3c deposition induced by COVID-19 patient serum as compared to the anti-C5 antibody. These results are supported by observations from case series of eculizumab, a monoclonal anti-C5 antibody, in which treated COVID-19 patients showed significant improvements in clinical parameters. ${ }^{12,13}$ However, the phase III trial of eculizumab (clinicaltrials gov. Identifier: NCT04355494) in COVID-19 patients on mechanical ventilation was paused due to interim analysis of 122 patients showing that the drug did not meet its prespecified efficacy outcome of survival on day 29. Final results from this trial are eagerly anticipated as are those for COVID-19 patients who are hospitalized but not on mechanical ventilation. COVID-19 patients may derive benefit from complement inhibition early in their disease course or from more proximal complement inhibition.

A comparative study of eculizumab versus AMY-101, an upstream C3 inhibitor, in a small number of patients showed that both decreased inflammatory markers and led to improvements in lung functions. The three patients who received AMY-101 demonstrated greater reduction in plasma levels of C3a, sC5b-9 and CFB as compared to patients who received eculizumab. ${ }^{37}$ This limited clinical data in addition to our in vitro results suggests that proximal complement inhibitors may be more effective than terminal inhibitors in reducing COVID-19 disease severity. Notably, treatment of six severely ill COVID-19 patients with Narsoplimab, a monoclonal antibody against MASP-2 inhibiting lectin-pathway activation, showed rapid reduction in serum inflammatory markers and survival in all patients. ${ }^{38}$ In addition to the COVID-19 infection itself, there are likely multifactorial contributions from tissue damage, secondary infections, and thrombosis, leading to complement activation from all pathways. Further studies comparing different complement inhibitors would be valuable to identify the most appropriate therapeutic targets.

The SARS-CoV-2 spike protein interferes with the function of CFH and this is likely an early event in the pathogenesis of COVID-19. In our prior work, we found that addition of purified $\mathrm{CFH}$ protein to serum treated with the SARS-CoV-2 spike protein decreased C3c and C5b-9 deposition on the cell surface. ${ }^{17}$ Here, we used competitive immunoprecipitation experiments to show that the SARSCoV-2 spike protein directly blocks CFH from binding to heparin, which may explain the APC dysregulation observed in COVID-19 infection (Figure 5). When binding to glycosaminoglycans on cell surface, including heparan sulfate, and $\alpha 2-3 \mathrm{~N}$-linked sialic acid residues, $\mathrm{CFH}$ achieves a more active conformation that allows for $\mathrm{C} 3 \mathrm{~b}$ binding. ${ }^{39}$ Interestingly, genetic variants in CFH, that occur in the same region where factor $\mathrm{H}$ binds heparan sulfate, have been identified as an important risk factor for morbidity and mortality from COVID-19. ${ }^{27}$

COVID-19 vaccines lead to the transient expression of the SARS-CoV-2 spike protein and are effective in preventing severe infection. ${ }^{40,41}$ Our vaccine studies (Figure 6) are reassuring that mRNA vaccines should not induce clinically significant complement amplification in healthy individuals, as suggested by the negative results from our functional assays; however, more data is necessary in patients with disorders of complement regulation, such as paroxysmal nocturnal hemoglobinuria (PNH), aHUS, CAPS, HELLP and cold agglutinin disease. ${ }^{42}$ Relapse of aHUS has been reported in patients with COVID-19 infection, ${ }^{43}$ and $\mathrm{PNH}$ patients have experienced adverse reactions to COVID-19 vaccines including severe hemolysis and need for blood transfusions even while on a C5 inhibitor. ${ }^{44}$ This evidence suggests that although complement activation induced by COVID-19 vaccines is wellcontrolled in healthy individuals, patients with disorders of complement regulation could be at higher risk for adverse reactions to vaccine.

Limitations of our study are that we received a limited amounts of patient serum for experiments and were unable to test other complement markers such as C4d deposition on the cell surface. We also had limited access to clinical information from which to draw robust conclusions regarding the association of complement activation with clinical parameters. Further, serum sample collection was not standardized and occurred at different time points from the initial diagnosis of COVID-19 and hospital admission. For example, in one of the two patients who died due to multiorgan failure from COVID-19, serum was collected near the end of his clinical course, at which point peak amplification of complement may have passed. Finally, we do not have serial samples from patients to estimate the persistence of complement activation over time. In future studies, it will be important to do serial mHam, Bb, and surface C5b-9 deposition studies starting soon after infection and correlating with SARS-CoV-2 viral load.

In summary, we showed that COVID-19 patient serum can induce complement dysregulation on cell surfaces that tracks with disease severity. Our previous data showed that the SARS-CoV-2 spike proteins convert inactivator surfaces to activator surfaces. Taken together, we postu- 
late that dysregulation of the APC is likely an early event after SARS-CoV-2 infection. Complement amplification from classical and lectin pathways following tissue damage, secondary infections, and thrombosis likely exacerbate end-organ damage similar to severe forms of aHUS and CAPS (Figure 7). Preconditions that lead to inflammation (e.g., obesity, diabetes and vascular disease) or contribute to complement activation (e.g., third-trimester pregnancy) or dysregulation (age-related macular degeneration and other germline complement mutations) may contribute to a severe phenotype. Indeed, components of the APC (CFB, CFD, and C3) are elevated in patients with obesity and insulin resistance..$^{45}$ Prospective studies correlating SARS-CoV-2 viral load to complement-mediated cell damage over the course of infection and additional genetic studies probing for rare variants in complement regulatory genes are needed. Our data also suggest that for complement inhibitors to be most effective, they should be initiated early in the disease process, but this too requires prospective study, as is the subject of the ongoing TACTIC-R study (clinical trials gov. Identifier: NCT04390464) ${ }^{46}$

\section{Disclosures}

$R A B$ has served on the advisory board for Alexion Pharmaceutical Inc.; SC has served on boards for Alexion and Sanofi-Genzyme, and her institution has received research funding on her behalf from Takeda.

\section{Contributions}

JY designed and performed experiments, analyzed the data, enrolled patients and wrote the first draft of the manuscript; GFG enrolled patients, analyzed the data, and edited the manuscript; $H C$ designed and performed the heparin binding assay, analyzed the data, and edited the manuscript; $X Y, S C$ and $E M B$ interpreted the data and edited the manuscript; $R A B$ designed the study, supervised the experiments, interpreted the data and wrote portions of the manuscript.

\section{Funding}

This work was supported by grants from National Institutes of Health (NIH), National Heart, Lung, and Blood Institute (NHLBI) grant RO1 HL 133113 (RAB); NIH grant KO8 HL138142 (EMB); NHLBI T32 HL 007525 (GFG).

\section{References}

1. Java A, Apicelli AJ, Liszewski MK, et al. The complement system in COVID-19: friend and foe? JCI Insight. 2020;5(15):e140711.

2. Gavriilaki E, Brodsky RA. Severe COVID19 infection and thrombotic microangiopathy: success does not come easily. $\mathrm{Br}$ J Haematol. 2020;189(6):e227-e230.

3. Risitano AM, Mastellos DC, Huber-Lang $\mathrm{M}$, et al. Complement as a target in COVID-19? Nat Rev Immunol. 2020; 20(6):343-344

4. Campbell CM, Kahwash R. Will complement inhibition be the new target in treating COVID-19-related systemic thrombosis? Circulation. 2020;141(22):1739-1741.

5. Chaturvedi S, Braunstein EM, Yuan X, et al. Complement activity and complement regulatory gene mutations are associated with thrombosis in APS and CAPS. Blood. 2020;135(4):239-251

6. Baines AC, Brodsky RA. Complementopathies. Blood Rev. 2017; 31(4):213-223

7. Wichmann D, Sperhake JP, Lütgehetmann $M$, et al. Autopsy findings and venous thromboembolism in patients with COVID-19: a prospective cohort study. Ann Intern Med. 2020;173(4):268-277.

8. Diao B, Wang C, Wang R, et al. Human kidney is a target for novel severe acute respiratory syndrome coronavirus 2 infection. Nat Commun. 2021;12(1):1-9

9. Carvelli J, Demaria O, Vély F, et al. Association of COVID-19 inflammation with activation of the C5a-C5aR1 axis. Nature. 2020;588(7836):146-150.

10. Cugno M, Meroni PL, Gualtierotti R, et al. Complement activation in patients with COVID-19: a novel therapeutic target. J Allergy Clin Immunol. 2020;146(1):215217.

11. de Nooijer AH, Grondman I, Janssen NAF, et al. Complement activation in the disease course of coronavirus disease 2019 and its effects on clinical outcomes. J Infect Dis. 2021;223(2):214-224

12. Diurno F, Numis FG, Porta G, et al.
Eculizumab treatment in patients with COVID-19: preliminary results from real life ASL Napoli 2 Nord experience. Eur Rev Med Pharmacol Sci. 2020;24(7):4040-4047.

13. Annane D, Heming N, Grimaldi-Bensouda L, et al. Eculizumab as an emergency treatment for adult patients with severe COVID-19 in the intensive care unit: A proof-of-concept study. EClinicalMedicine. 2020;28:100590

14. Mastaglio S, Ruggeri A, Risitano AM, et al. The first case of COVID-19 treated with the complement C3 inhibitor AMY-101. Clin Immunol. 2020;215:108450.

15. Ling M, Murali M. Analysis of the complement system in the clinical immunology laboratory. Clin Lab Med. 2019;39(4):579590.

16. Kourtzelis I, Markiewski MM, Doumas M, et al. Complement anaphylatoxin C5a contributes to hemodialysis-associated thrombosis. Blood. 2010;116(4):631-639.

17. Yu J, Yuan X, Chen H, Chaturvedi S, Braunstein EM, Brodsky RA. Direct activation of the alternative complement pathway by SARS-CoV-2 spike proteins is blocked by factor D Inhibition. Blood. 2020;136(18):2080-2089.

18. Gavriilaki E, Yuan X, Ye Z, et al. Modified Ham test for atypical hemolytic uremic syndrome. Blood. 2015;125(23):3637-3646.

19. Vaught AJ, Braunstein EM, Jasem J, et al. Germline mutations in the alternative pathway of complement predispose to HELLP syndrome. JCI Insight. 2018;3(6):5-7.

20. World Health Organization. COVID-19 Therapeutic Trial Synopsis. World Health Organization. https://cdn. who.int/media/ docs/default-source/blue-print/covid-19the rapeutic-trial-synopsis. pdf?.sfvrsn $=44$ b83344_1\&download $=$ true 2020, Accessed May 12, 2021.

21. Yuan X, Yu J, Gerber G, et al. Ex vivo assays to detect complement activation in complementopathies. Clin Immunol. 2020; 221: 108616.

22. Vaught AJ, Braunstein EM, Jasem J, et al. Germline mutations in the alternative pathway of complement predispose to HELLP syndrome. JCI Insight. 2018;3(6):e99128.

23. Clausen TM, Sandoval DR, Spliid CB, et al.
SARS-CoV-2 infection depends on cellular heparan sulfate and ACE2. Cell. 2020;183(4):1043-1057.e15

24. Magro C, Mulvey JJ, Berlin D, et al. Complement associated microvascular injury and thrombosis in the pathogenesis of severe COVID-19 infection: a report of five cases. Transl Res. 2020;220:1-13.

25. Holter JC, Pischke SE, de Boer E, et al Systemic complement activation is associated with respiratory failure in COVID-19 hospitalized patients. Proc Natl Acad Sci U S A. 2020;117(40):25018-25025.

26. Sinkovits G, Mező B, Réti $M$, et al. Complement overactivation and consumption predicts in-hospital mortality in SARS CoV-2 infection. Front Immunol. 2021;12 663187.

27. Ramlall V, Thangaraj PM, Meydan C, et al. Immune complement and coagulation dysfunction in adverse outcomes of SARS CoV-2 infection. Nat Med. 2020; 26(10):1609-1615.

28. Valenti L, Griffini S, Lamorte G, et al. Chromosome 3 cluster rs11385942 variant links complement activation with severe COVID-19. J Autoimmun. 2021;117: 102595.

29. Gavriilaki E, Asteris PG, Touloumenidou T, et al. Genetic justification of severe COVID-19 using a rigorous algorithm. Clin Immunol. 2021;226:108726.

30. Jang S, Rhee JY, Wi YM, Jung BK. Viral kinetics of SARS-CoV-2 over the preclinical, clinical, and postclinical period. Int J Infect Dis. 2021;102:561-565.

31. Sun J, Tang X, Bai R, et al. The kinetics of viral load and antibodies to SARS-CoV-2. Clin Microbiol Infect. 2020;26(12):1690.e11690.e4.

32. Messner CB, Demichev V, Wendisch D, et al. Ultra-high-throughput clinical proteomics reveals classifiers of COVID-19 infection. Cell Syst. 2020;11(1):11-24.e4.

33. Yan B, Freiwald T, Chauss D, et al. SARSCoV-2 drives JAK1/2-dependent local complement hyperactivation. Sci Immunol. 2021;6(58):1-20.

34. Macor P, Durigutto P, Mangogna A, et al. Multi-organ complement deposition in COVID-19 patients. medRxiv. 2021 Jan 8. 
doi: $\quad$ 10.1101/2021.01.07.21249116 [preprint, not peer-reviewed].

35. Pekayvaz K, Leunig A, Kaiser R, et al. Protective immune trajectories in early viral containment of non-pneumonic SARSCoV-2 infection. bioRxiv. doi: https://doi.org/10.1101/2021.02.03.429351 [preprint, not peer-reviewed].

36. Ma L, Sahu SK, Cano M, et al. Increased complement activation is a distinctive feature of severe SARS-CoV-2 infection. Sci Immunol. 2021;6(59):1-18.

37. Mastellos DC, Pires da Silva BGP, Fonseca BAL, et al. Complement C3 vs C5 inhibition in severe COVID-19: early clinical findings reveal differential biological efficacy. Clin Immunol. 2020;220:108598.

38. Rambaldi A, Gritti G, Micò MC, et al. Endothelial injury and thrombotic microan- giopathy in COVID-19: treatment with the lectin-pathway inhibitor narsoplimab. Immunobiology. 2020;225(6):152001.

39. Perkins SJ, Fung KW, Khan S. Molecular interactions between complement factor $\mathrm{H}$ and its heparin and heparan sulfate ligands. Front Immunol. 2014;5:1-14.

40. Polack FP, Thomas SJ, Kitchin N, et al. Safety and Efficacy of the BNT162b2 mRNA Covid-19 Vaccine. N Engl J Med. 2020;383(27):2603-2615.

41. Baden LR, El Sahly HM, Essink B, et al. Efficacy and safety of the mRNA-1273 SARS-CoV-2 Vaccine. N Engl J Med. 2021;384(5):403-416.

42. Gavriilaki E， Brodsky RA Complementopathies and precision medicine. J Clin Invest. 2020;130(5):2152-2163.

43. Ville S, Le Bot S, Chapelet-Debout A, et al.
Atypical HUS relapse triggered by COVID19. Kidney Int. 2021;99(1):267-268

44. Gerber GF, Yuan X, Yu J, et al. COVID-19 Vaccines induce severe hemolysis in paroxysmal nocturnal hemoglobinuria. Blood. 2021;137(26):3670-3673.

45. Shim K, Begum R, Yang C, Wang $H$. Complement activation in obesity, insulin resistance, and type 2 diabetes mellitus. World J Diabetes. 2020;11(1):1-12.

46. Kulkarni S, Fisk M, Kostapanos M, et al. Repurposed immunomodulatory drugs for Covid-19 in pre-ICU patients - multi-arm Therapeutic study in pre-ICU patients admitted with Covid-19- repurposed drugs (TACTIC-R): a structured summary of a study protocol for a randomised controlled trial. Trials. 2020;21 (1):20-22. 